\title{
Aktuelle Mindestlohndebatte: Branchenlösungen oder gesetzlicher Mindestlohn?
}

Der Niedriglohnsektor ist in Deutschland in den letzten Jahren immer größer geworden - einer der Hauptgründe für „Armut trotz Arbeit“. Dies hat zu einer intensiven politischen Auseinandersetzung um die Einführung von Mindestlöhnen geführt. Die Bundesregierung plant, mit der Ausweitung des Arbeitnehmer-Entsendegesetzes und einer Novellierung des Mindestarbeitsbedingungengesetzes die Möglichkeiten zur Einführung branchenbezogener Mindestlöhne zu erleichtern. Eine genaue Analyse der vorgeschlagenen Verfahren macht deutlich, dass damit große Teile des Niedriglohnsektors nicht erfasst werden würden. Deshalb bleibt der allgemeine, branchenübergreifende Mindestlohn auf der Tagesordnung.

\section{Bestehende Mindestlohn- regelungen}

Seit Mitte der 1990er Jahre hat die Anzahl der Niedriglohnempfänger in Deutschland kontinuierlich zugenommen. Legt man als Niedriglohnschwelle die OECD-Definition von zwei Dritteln des Medianlohnes zugrunde, so waren im Jahr 2006 knapp 6,6 Mio. Beschäftigte oder 22,6\% aller Beschäftigten im Niedriglohnsektor tätig (Kalina/Weinkopf 2008). Vor diesem Hintergrund findet seit einigen Jahren auch in Deutschland eine kontroverse Debatte über die Einführung eines gesetzlichen Mindestlohns statt. Denn im Gegensatz zur Mehrzahl der europäischen Staaten existiert hierzulande bislang kein allgemeiner, branchenübergreifender gesetzlicher Mindestlohn. Stattdessen gibt es lediglich verschiedene Formen von branchenbezogenen tariflichen Mindestlöhnen, die eine jeweils unterschiedliche Reichweite und Verbindlichkeit aufweisen. Drei Formen von tariflichen Mindestlöhnen können unterschieden werden:

\subsection{TARIFLÖHNE ALS MINDESTLÖHNE}

Die in Tarifverträgen vereinbarten Löhne sind Mindestvergütungen, die nicht unterschritten werden dürfen. Allerdings gelten diese Tarifvergütungen nur für die Mitglieder der jeweils vertragschließenden Tarifparteien, also die Gewerkschaftsmitglieder und die Mitglieder des Arbeitgeberverbandes bzw. des Unternehmens, das die Tarif-

\begin{tabular}{|c|c|c|c|}
\hline Tarifbereich & Personengruppe & $€ /$ Stunde & $€ /$ Monat \\
\hline Bekleidungsindustrie Niedersachsen/Bremen & Angestellte/r & 6,32 & 1.011 \\
\hline $\begin{array}{l}\text { Bewachungsgewerbe Thüringen } \\
\text { Revierwachdienst }\end{array}$ & Arbeiter/in & 4,75 & 823 \\
\hline Einzelhandel Mecklenburg-Vorpommern & Arbeiter/in & 6,78 & 1.146 \\
\hline Erwerbsgartenbau Brandenburg & Arbeiter/in & 4,71 & 857 \\
\hline Fleischerhandwerk Thüringen & Arbeiter/in & 5,49 & 928 \\
\hline Floristik West & Arbeitnehmer/in & 5,94 & 1.004 \\
\hline Friseurhandwerk Baden-Württemberg & Arbeitnehmer/in & 6,38 & 1.027 \\
\hline $\begin{array}{l}\text { Gebäudereinigerhandwerk Brandenburg-Ost, } \\
\text { Potsdam }\end{array}$ & Arbeiter/in & 6,36 & 1.074 \\
\hline Hotel- und Gaststättengewerbe Saarland & Arbeitnehmer/in & 6,27 & 1.085 \\
\hline Landwirtschaft Niedersachsen/Weser-Ems & Arbeiter/in & 5,98 & 1.041 \\
\hline Metallhandwerk Brandenburg & Arbeiter/in & 7,12 & 1.146 \\
\hline Öffentlicher Dienst Gemeinden Ost & Arbeitnehmer/in & 7,20 & 1.247 \\
\hline Privates Transport- u. Verkehrsgewerbe Thüringen & Arbeitnehmer/in & 5,12 & 886 \\
\hline Systemgastronomie Ost o. Berlin-Ost & Arbeitnehmer/in & 6,14 & 1.050 \\
\hline Zeitarbeit Bundesverband Zeitarbeit (BZA) West & Arbeitnehmer/in & 7,38 & 1.119 \\
\hline
\end{tabular}

vereinbarungen getroffen hat. Beschäftigte in nicht tarifgebundenen Unternehmen haben keinen Anspruch auf die Tarifvergütungen. Es ist jedoch eine weitverbreitete betriebliche Praxis, durch arbeitsvertragliche Bezugnahme auch für die nicht gewerkschaftlich organisierten Beschäftigten die Anwendung der Tarifverträge sicherzustellen. Auf dieser Basis ergibt sich eine Tarifbindung von rund $63 \%$ der Beschäftigten und rund $37 \%$ der Betriebe. $40 \%$ der nicht tarifgebundenden Betriebe geben an, sich am Tarifvertrag zu orientieren, was jedoch keineswegs eine vollständige Umsetzung der tariflichen Vorschriften bedeutet.

Eine Analyse der tariflichen Lohn- und Gehaltsstrukturen zeigt, dass die tarifliche Vergütungsregelung keinesfalls als sicheres Instrument zur Vermeidung von Niedriglöhnen angesehen werden kann. In zahlrei- chen Branchen bestehen tarifliche Grundvergütungen, die deutlich unterhalb der aktuell diskutierten Höhe von 7,50€ pro Stunde für einen möglichen gesetzlichen Mindestlohn liegen (Bispinck/WSI-Tarifarchiv 2007). Dies gilt in besonderem Maße, aber keineswegs ausschließlich, für ostdeutsche Tarifgebiete (Tabelle 1). Betroffen sind auch nicht nur die jeweils unters-

Reinhard Bispinck, Dr., Wissenschaftler im WSI und Leiter des WSI-Tarifarchivs in der Hans-Böckler-Stiftung.

e-mail:Reinhard-Bispinck@boeckler.de Thorsten Schulten, Dr., Wissenschaftler im WSI in der Hans-Böckler-Stiftung. Arbeitsschwerpunkte: Arbeits- und Tarifpolitik in Europa. e-mail: Thorsten-Schulten@boeckler.de 
ten Tarifgruppen, vielmehr reichen die tariflichen Niedriglöhne in einigen Wirtschaftszweigen bis weit in die mittleren Lohn- und Gehaltsgruppen hinein (z. B. Wach- und Sicherheitsgewerbe, Hotels und Gaststätten, Friseurgewerbe).

\subsection{ALLGEMEINVERBINDLICH ERKLÄRTE TARIFLÖHNE}

Tarifverträge können vom Bundesarbeitsminister im Einvernehmen mit dem paritätisch besetzten Tarifausschuss auf Antrag einer Tarifpartei für allgemeinverbindlich erklärt werden. Sie erlangen dadurch Gültigkeit auch für alle nicht tarifgebundenen Arbeitgeber und Beschäftigten des tariflichen Geltungsbereichs. Voraussetzung für die Allgemeinverbindlicherklärung (AVE) ist, dass die tarifgebundenen Arbeitgeber nicht weniger als $50 \%$ der unter den Geltungsbereich fallenden Arbeitnehmerinnen und Arbeitnehmer beschäftigen und ein öffentliches Interesse an der AVE besteht. Allgemeinverbindliche Vergütungstarifverträge gibt es lediglich in einigen regionalen Tarifbereichen des Friseurgewerbes, des Wach- und Sicherheitsgewerbes, des Hotel- und Gaststättengewerbes sowie des gesamten Gebäudereinigerhandwerkes. Davon werden insgesamt rund 570.000 Beschäftigte erfasst.

\subsection{TARIFLICHE MINDESTLÖHNE NACH DEM ENTSENDEGESETZ}

Das Arbeitnehmer-Entsendegesetz (AEntG) von 1996 schreibt vor, dass die Rechtsnormen eines für allgemeinverbindlich erklärten Tarifvertrages des Baugewerbes auch für solche Arbeitsverhältnisse zwingend angewendet werden müssen, die zwischen einem ausländischen Unternehmen und seinen in Deutschland beschäftigten Arbeitnehmerinnen und Arbeitnehmern bestehen. Im vergangenen Jahr wurde das AEntG auch auf das Gebäudereinigerhandwerk (Juli 2007) und auf die Briefdienstleistungen (Januar 2008) ausgeweitet.

Aktuell gibt es in sieben Wirtschaftszweigen tarifliche Mindestlöhne, die nach dem AEntG für allgemeinverbindlich erklärt wurden:

- Bauhauptgewerbe (438.300 Beschäftigte),

- Maler- und Lackiererhandwerk (109.000 Beschäftigte),

\begin{tabular}{|c|c|c|c|c|}
\hline $\begin{array}{l}\text { Übersicht 1: Tarifliche } \\
\text { Entsendegesetz (AEnt }\end{array}$ & $\begin{array}{l}\text { Mindestlöhn } \\
\text { i) - in €/Stunc }\end{array}$ & nach dem & Arbeitnehr & \\
\hline Geltende Verträge & & & & \\
\hline Abbruch- u. Abwrackgewerbe 1 & & $a b 09 / 2007$ & & \\
\hline West & Hilfskraft & 9,79 & & \\
\hline & Fachwerker & 11,96 & & \\
\hline Ost & Hilfskraft & 9,10 & & \\
\hline & Fachwerker & 10,16 & & \\
\hline Bauhauptgewerbe & & $a b 09 / 2007$ & $a b 09 / 2008$ & \\
\hline West inkl. Berlin & Werker & 10,40 & 10,70 & \\
\hline & Fachwerker & 12,50 & $\begin{array}{c}12,85 \\
\text { (Berlin: } 12,70)\end{array}$ & \\
\hline Ost & Werker & 9,00 & & \\
\hline & Fachwerker & 9,80 & & \\
\hline Briefdienstleistungen & & $a b 01 / 2008$ & $a b 01 / 2010$ & \\
\hline West inkl. Berlin & Briefzusteller & 9,80 & & \\
\hline & $\begin{array}{l}\text { sonstige } \\
\text { Tätigkeiten }\end{array}$ & 8,40 & & \\
\hline Ost & Briefzusteller & 9,00 & 9,80 & \\
\hline & $\begin{array}{l}\text { sonstige } \\
\text { Tätigkeiten }\end{array}$ & 8,00 & 8,40 & \\
\hline Dachdeckerhandwerk & & $a b 01 / 2008$ & $a b 01 / 2009$ & \\
\hline West und Ost & Mindestlohn & 10,20 & 10,40 & \\
\hline Elektrohandwerk (Montage) & & $a b 01 / 2008$ & $a b 01 / 2009$ & $a b 01 / 2010$ \\
\hline West & Mindestentgelt & 9,40 & 9,55 & 9,60 \\
\hline Ost inkl. Berlin & Mindestentgelt & 7,90 & 8,05 & 8,20 \\
\hline Gebäudereinigerhandwerk & & $a b 07 / 2007$ & $a b 03 / 2008$ & \\
\hline West inkl. Berlin & unterste Lohngr. & 7,87 & 8,15 & \\
\hline Ost & unterste Lohngr. & 6,36 & 6,58 & \\
\hline Maler- u. Lackiererhandwerk & & $a b 04 / 2008$ & & \\
\hline West & ungelernter AN & 8,05 & & \\
\hline & Geselle & 11,05 & & \\
\hline Ost & ungelernter AN & 7,50 & & \\
\hline & Geselle & 9,65 & & \\
\hline Abgeschlossen, aber noch nicht & Is AEntG aufgenon & en: & & \\
\hline Industrielle textile Dienste ${ }^{2}$ & & ab $11 / 2007$ & $a b 03 / 2008$ & \\
\hline West & Mindestentgelt & $\begin{array}{c}9,02 \\
\text { ab } 06 / 2007\end{array}$ & 9,20 & \\
\hline Ost & Mindestentgelt & 7,83 & 8,01 & \\
\hline Zeitarbeit (BZA, iGZ) ${ }^{2}$ & & $a b 01 / 2007$ & $a b 01 / 2008$ & \\
\hline West & Mindestentgelt & 7,15 & 7,31 & \\
\hline Ost inkl. Berlin & Mindestentgelt & 6,22 & 6,36 & \\
\hline $\begin{array}{l}\text { 1) Antrag auf Allgemeinverbindlicherk } \\
\text { 2) Einbeziehung der Branche in das } A E\end{array}$ & $\begin{array}{l}\text { (AVE) gestellt, alte } A \\
\text { och nicht erfolgt. }\end{array}$ & nde 08/2007 a & aufen. & \\
\hline Quelle: WSI-Tarifarchiv, Stand: 29.02 .20 & & & & EILUNGEN \\
\hline
\end{tabular}

- Dachdeckerhandwerk (58.600 Beschäftigte),

- Abbruchgewerbe (9.700 Beschäftigte),

- Gebäudereinigerhandwerk (321.200 Beschäftigte),

- Elektrohandwerk (278.000 Beschäftigte),

- Briefdienstleistungen (140.000 Beschäftigte).

Damit besteht in Branchen mit rund 1,3 Mio. Beschäftigten ein tariflicher Mindestlohn nach dem Arbeitnehmer-Entsendegesetz (AEntG). Die Höhe dieser Mindestlöhne schwankt je nach Branche und Region zwischen 6,36€ und 12,50€(Übersicht 1). In einigen Tarifbereichen sind gestaffelt nach Qualifikation bzw. Tätigkeit zwei Mindestlöhne vorgesehen. In der Zeitarbeitsbranche und bei den industriellen textilen Diensten sind bereits Mindestlohntarifverträge abgeschlossen, die noch nicht in das AEntG aufgenommen wurden.

\section{Neue Vorschläge für brachenbezogene Mindestlöhne}

Nach monatelangen Kontroversen einigte sich die Große Koalition im Juni 2007 auf 


\section{Übersicht 2: Vereinbarte bzw. angestrebte Mindestlohntarifverträge nach AEntG}

- Zeitarbeit: Seit Juni 2006 liegt ein Tarifvertrag Mindestlohn vor, der zwischen der DGB-Tarifgemeinschaft und den beiden Verbänden Bundesverband Zeitarbeit (BZA) und Interessenverband Deutscher Zeitarbeitsunternehmen (iGZ) abgeschlossen wurde. Die AVE scheiterte bislang daran, dass es konkurrierende Verträge gibt, insbesondere zwischen der Tarifgemeinschaft Christlicher Gewerkschaften Zeitarbeit und PSA (CGZP) und der Arbeitsgemeinschaft Mittelständischer Personaldienstleister (AMP), die im Niveau darunter liegen.

- Industrielle textile Dienste: Die IG Metall hat im Herbst 2007 einen Vertrag mit dem Industrieverband Textil Service intex e. V. abgeschlossen. Er sieht Mindestlöhne von 9,20/8,01 € (West/Ost) ab dem 1.3.2008 für Betriebe ab 20 Beschäftigten vor.

- Forstwirtschafliche Dienstleistungen: Auch für diesen Tarifbereich mit rund 10.000 Beschäftigten liegt bereits ein von der IG BAU ausgehandelter Mindestlohntarifvertrag vor. Er beinhaltet einen Mindestlohn von 8,50€, der bis zum 1.7.2009 in zwei Stufen auf 10,26€ steigen soll.

- Weiterbildung: GEW und ver.di haben mit dem Bundesverband berufliche Bildung (BVB) im März 2007 einen Mindestlohntarifvertrag für den Bereich der außerbetrieblichen Qualifizierung und der sozialen Integration abgeschlossen. Er sieht für Verwaltungsangestellte ein Mindestgehalt von $1.809,33 / 1.610,84 €$ (West/Ost) und für pädagogische Mitarbeiter ein Mindestgehalt von 2.076,06/1.847,69 € (West/Ost) vor. Zurzeit wird der Geltungsbereich des Vertrages präzisiert.

- Wach- und Sicherheitsgewerbe: Die Verhandlungen zwischen ver.di und dem Bundesverband Deutscher Wach- und Sicherheitsunternehmen (BDWS) scheiterten, weil die Arbeitgeber sich nicht in der Lage sahen, für alle westlichen Bundesländer einen Mindestlohn über 7,50€ zu vereinbaren. Auch für den Osten waren die angebotenen Entgelte aus Gewerkschaftssicht zu niedrig. Der Arbeitgeberverband hat daraufhin Verhandlungen mit dem Christlichen Gewerkschaftsbund aufgenommen.

- Entsorgungswirtschaft: Die Tarifparteien streben einen Mindestlohn an. Keine Einigung besteht über die von ver.di geforderten Beträge von 10,50 bis $11 €$. Über eine Zuordnung von Tätigkeiten wurde noch nicht gesprochen. In der Branche arbeiten rund 90.000 Beschäftigte im kommunalen Bereich und 70.000 in privaten Betrieben, von denen die Hälfte tariflich entlohnt wird.

- Gartenbau: Beide Tarifparteien, die IG BAU und der Zentralverband Gartenbau, sind bereit, Mindestlöhne für diesen Tarifbereich zu vereinbaren. Konkrete Gesprächsergebnisse liegen noch nicht vor.

- Schienenverkehr: Die Tarifgemeinschaft der Gewerkschaften Transnet und der Gewerkschaft Deutscher Bundesbahnbeamter, Arbeiter und Angestellter (GBDA) will für diese Branche einen möglichst umfassenden Mindestlohntarifvertrag vereinbaren, der drei verschiedene Lohnniveaus integriert (unterster Mindestlohn, einfache Facharbeiten, qualifizierte Facharbeiten).

- Hotel- und Gaststättengewerbe: Die Gewerkschaft NGG strebt eine tarifliche Regelung nach dem AEntG an, aber der Arbeitgeberverband Deutscher Hotel- und Gaststättenverband (DEHOGA) lehnt einen Mindestlohn grundsätzlich ab. In einigen regionalen Tarifbereichen bestehen seit geraumer Zeit tariflose Zustände. Das Tarifniveau liegt in der untersten Gruppe zum Teil deutlich unter $6 €$ in der Stunde.

- Ernährungshandwerk: In diesem Bereich werden seitens der Gewerkschaft NGG Gespräche mit den Arbeitgebern geführt, um gegebenenfalls untere Lohngrenzen zu vereinbaren. Ob dies in Form von Mindestlöhnen nach dem Entsendegesetz umgesetzt werden kann, ist derzeit noch offen.

- Fleischindustrie: Die Tarifbindung in diesem Bereich ist sehr gering, zudem ist eine Bereitschaft zur Vereinbarung bundesweiter Mindestlöhne auf Arbeitgeberseite kaum vorhanden.

- Pflegebereich: Im Bereich der ambulanten und stationären (Alten-)Pflege strebt ver.di mit den Wohlfahrtsverbänden und privaten Trägern ebenfalls einen entsprechenden Tarifvertrag an. Hier gibt es u.a. Probleme mit den kirchlichen Trägern, die nicht unter das Tarifrecht fallen.

- Friseurhandwerk: Grundsätzlich ist ver.di an einer tariflichen Mindestlohnregelung interessiert, eine Einigung auf einen Mindestlohn von 7,50 € erscheint aber angesichts der $z$. T. extrem niedrigen tariflichen Grundvergütungen derzeit völlig aussichtslos.

- Einzelhandel: Ver.di ist an einem Mindestlohn-Tarifvertrag grundsätzlich interessiert. Der Hauptverband des Deutschen Einzelhandels (HDE) lehnt jedoch in Wirtschaftszweigen mit Flächentarifverträgen gesetzlich festgelegte Mindestlöhne $a b$.

einen Minimalkompromiss in Sachen Mindestlohn. Dieser eröffnet allen Branchen, in denen mindestens $50 \%$ der Beschäftigten tarifgebunden sind, die Möglichkeit, in das Arbeitnehmer-Entsendegesetz aufgenommen zu werden. Für die anderen Branchen soll ein Mindestlohn auf der Grundlage des zu modernisierenden Mindestarbeitsbedingungengesetzes von 1952 festgesetzt werden können. Anfang Januar 2008 legte Bundesarbeitsminister Scholz die Referentenentwürfe zur Novellierung des Arbeitnehmerentsendegesetzes und des Mindestarbeitsbedingungengesetzes vor (BMAS 2008a, 2008b). ${ }^{1}$

\subsection{AUSWEITUNG DES ENTSENDEGESETZES}

Das Entsendegesetz soll - so heißt es in der Entwurfsbegründung - der sozialen Flankierung des europäischen Binnenmarktes dienen $u n d$ angesichts zurückgehender Tarifbindung die Einhaltung von Mindeststandards im Entgeltbereich mit gesetzlichen Maßnahmen flankieren. Der Gesetzentwurf sieht dazu folgende Regelungen vor (BMAS 2008a): Voraussetzung für die Aufnahme von Branchen in das AEntG ist eine Tarifbindung von insgesamt mindestens $50 \%$ der Beschäftigten. Die Tarifver- tragsparteien einzelner Branchen können bis Ende März 2008 ihr Interesse bekunden. Auch danach ist eine Aufnahme in das AEntG möglich. Die Allgemeinverbindlichkeit nach dem AEntG kann durch Rechtsverordnung des BMAS ohne $\mathrm{Zu}-$ stimmung des Bundesrates bestimmt werden. Wenn eine Branche erstmals einen entsprechenden Antrag stellt, wird ein paritätisch besetzter Bundestarifausschuss damit befasst. Künftig sollen auch Tarifverträge, die lediglich regionale Geltung haben, nach dem AEntG allgemeinverbindlich erklärt werden können, was nach dem bisherigen Gesetzeswortlaut nicht möglich war. Bei konkurrierenden Tarifverträgen soll die Zahl der jeweils erfassten Beschäftigten und Gewerkschaftsmitglieder als Entscheidungskriterium herangezogen werden. Ferner soll das fiskalische Interesse an einem ausreichenden Erwerbseinkommen ohne notwendige Aufstockung durch Grundsicherung für Arbeitsuchende berücksichtigt werden.

Die Tarifvertragsparteien prüfen zurzeit, ob und unter welchen Bedingungen sie in einzelnen Branchen Mindestlohn-Tarifverträge abschließen können. Übersicht 2 zeigt, welche Branchen in der Diskussion sind und welche bereits Tarifverträge abgeschlossen haben.

Grundsätzlich liegen die Stärken und Schwächen von Mindestlöhnen nach dem Arbeitnehmerentsendegesetz (AEntG) auf der Hand: In Branchen mit funktionierender Tarifvertragspraxis auf der Basis von Flächentarifverträgen können Mindestlohn-Tarifverträge nach dem AEntG akzeptable Mindeststandards definieren. Damit kann - bei geeigneter Umsetzung und Kontrolle - ein Lohndumping durch nicht tarifgebundene Außenseiter aus dem In- und Ausland innerhalb der Branche unterbunden werden. Zugleich kann der Gefahr begegnet werden, dass ein niedriger branchenübergreifender Mindestlohn als negativer Referenzpunkt für die unterste Tarifvergütung einer Branche herangezogen wird.

Eine begrenzte Wirkung erzielt das AEntG, wenn die tariflichen Grundvergütungen der regulären Tarifverträge sehr niedrig ausfallen. Eine Allgemeinverbind-

1 Die Gesetzentwürfe stießen auf Widerspruch des Bundeskanzleramtes und des Bundeswirtschaftsministeriums. Zum Zeitpunkt der Abfassung dieses Artikels lag noch keine abgestimmte Fassung der Entwürfe vor. 
licherklärung von tariflichen Niedriglöhnen mag zwar deren weitere Unterschreitung verhindern, löst aber das zugrunde liegende Kernproblem unzureichender Vergütung nicht. Gar keine Wirkung entfaltet das Entsendegesetz in Bereichen ohne einschlägige Tarifverträge bzw. mit geringer Tarifbindung.

Damit beschränkt sich die Wirkung des Entsendegesetzes auf die Re-Stabilisierung von angemessenen Einkommensstandards in relativ gut tarifierten Branchen. Die genannte Liste von Tarifbereichen, die für das AEntG eventuell infrage kommen, zeigt, dass durchaus ein breiteres Anwendungspotenzial vorhanden ist, allerdings werden zugleich auch die systematischen Leerstellen der AEntG-Strategie deutlich. In jedem Fall hat die Arbeitgeberseite eine starke Veto-Position, die ihr ermöglicht, durch die Verweigerung eines Mindestlohntarifvertrags das gesamte Regelungssystem zu unterlaufen.

\subsection{REAKTIVIERUNG DES MINDEST- ARBEITSBEDINGUNGENGESETZES}

Die vom Koalitionsausschuss beschlossene Aktualisierung des Mindestarbeitsbedingungengesetzes von 1952 soll das Gesetz vor allem in Bereichen mit geringer Tarifbindung (unter $50 \%$ ) bzw. ohne tarifvertragliche Regelungen anwendbar machen (BMAS 2008b). Der Referentenentwurf sieht einen siebenköpfigen Hauptausschuss vor, der darüber entscheiden soll, $o b$ für Wirtschaftszweige bundesweit oder regional Mindestarbeitsbedingungen festgesetzt werden sollen. Der Hauptausschuss soll mit unabhängigen Fachleuten besetzt werden, die Spitzenverbände haben dazu ein Vorschlagsrecht. Die konkrete Ausgestaltung der Mindestarbeitsbedingungen soll von Fachausschüssen vorgenommen werden, die sich paritätisch aus Vertretern der Arbeitnehmer- und Arbeitgeberseite zusammensetzen. Die Bundesregierung soll dann die Mindestarbeitsbedingungen durch Rechtsverordnung erlassen. Als Kriterien für die Arbeit der Fachausschüsse werden genannt:

- Schaffung angemessener Arbeitsbedingungen,

- Gewährleistung fairer Wettbewerbsbedingungen der Unternehmen,

- Erhaltung sozialversicherungspflichtiger Beschäftigung, Bekämpfung von Arbeitslosigkeit,
- fiskalische Interessen, sodass bei Vollzeitarbeit keine regelmäßigen Leistungen aus der Grundsicherung erfolgen müssen, - Berücksichtigung von Auswirkungen auf bestehende Tarifverträge.

Praktische Erfahrungen mit dem Mindestarbeitsbedingungengesetz liegen nicht vor, der einzige Anwendungsfall aus dem Jahr 1952 für Beschäftigte bei Rechtsanwälten und Notaren wurde nicht zu Ende geführt (Peter 2004). Die geplanten Rahmenund Ausführungsbestimmungen lassen befürchten, dass die Anwendung des Gesetzes sehr bürokratisch und zeitaufwendig sein wird. Mögliche Hürden sind:

(1) Der Branchenbezug: Regelungen können immer nur auf bestimmte Branchen bezogen werden. Dies hätte allenfalls dann Vorteile, wenn die Standards deutlich oberhalb eines bestimmten Mindestniveaus festgesetzt würden, wie dies bei einigen Branchenregelungen nach dem AEntG tatsächlich der Fall ist. Wenn es aber im Kern um die Etablierung eines unteren Lohnniveaus geht, wie es auch für einen branchenübergreifenden Mindestlohn in der Debatte ist, macht ein solcher Branchenbezug wenig Sinn. Zu erwarten ist auch, dass zahlreiche Lücken bleiben, wenn nicht systematisch und flächendeckend für alle in Betracht kommenden Niedriglohnbranchen Verfahren in Gang gesetzt werden.

(2) Verfahrenshürden: Für jede Branche muss ein zweistufiges Verfahren durchlaufen werden. Erst nach Bewilligung durch den Hauptausschuss kann sich der Fachausschuss mit der konkreten Regulierung befassen. Für jedes Verfahren hat der Hauptausschuss sechs Monate Zeit. Insbesondere in der Anfangsphase dürfte der Hauptausschuss tendenziell überlastet sein. Angesichts der problematischen Erfahrungen mit den klassischen AVE-Verfahren ist damit zu rechnen, dass die Verfahren vielfach ohne konkretes Ergebnis enden.

(3) Kontrolle und Umsetzung: Bereits jetzt ist die Regelungs- und Informationslage bei den tariflichen Mindestlöhnen nach dem Entsendegesetz unübersichtlich. Wenn nach dem Mindestarbeitsbedingungengesetz zahlreiche neue Mindestregelungen hinzukommen, ergibt sich ein Flickenteppich von sektoral und regional unterschiedlichen Mindestlohnniveaus, die schwer zu vermitteln und noch schwerer zu kontrollieren sind.

(4) Die Anpassung der Mindeststandards: Wenn das materielle Sicherungsniveau der Mindeststandards erhalten bleiben soll, ist eine regelmäßige kurzfristige Anpassung erforderlich. Bei dem jetzt vorgesehenen Verfahren ist nicht ersichtlich, wie dies in akzeptablen Zeiträumen geschehen soll.

\section{Die universelle Losung: \\ Die universelle Lösung: Ein branchenüber- greifender Mindestlohn}

Der fragile und bürokratische Charakter branchenbezogener Mindestlohnregelungen und die hierbei nicht vermeidbaren beachtlichen Regelungslücken werden auch in Deutschland dafür sorgen, dass eine universelle Lösung im Sinne eines allgemeinen, branchenübergreifenden Mindestlohns auf der Tagesordnung bleibt. Nicht zufällig haben sich nahezu sämtliche EUStaaten mit gesetzlichen Mindestlöhnen deshalb für ein universelles, branchenübergreifendes Mindestlohnmodell entschieden (Schulten et al. 2006).

Aus den Erfahrungen anderer europäischer Länder lassen sich für die Umsetzung eines allgemeinen Mindestlohns folgende Grundprinzipien ableiten (ebd, S. 16ff.): Entgegen einer weitverbreiteten Vorstellung ist es in der Regel nicht einfach der Staat, der die Höhe des gesetzlichen Mindestlohns bestimmt. Die konkrete Festlegung und Anpassung erfolgt vielmehr in enger Kooperation mit Gewerkschaften und Arbeitgeberverbänden. Letztere werden über bi- und tripartistische Beratungsgremien in die Ausgestaltung der Mindestlohnpolitik institutionell eingebunden. Ein besonderer Ansatz wurde in Großbritannien entwickelt, wo eine drittelparitätisch aus Arbeitgebern, Gewerkschaften und Wissenschaft zusammengesetzte Low Pay Commission auf der Grundlage umfangreicher Studien jährlich Empfehlungen zur Erhöhung des Mindestlohns ausspricht (Burgess 2006).

In zahlreichen europäischen Ländern gibt es außerdem Verfahrensregeln zur regelmäßigen Anpassung des gesetzlichen Mindestlohns. So haben einige Länder einen bestimmten Stichtag festgelegt, an dem 
- meistens einmal pro Jahr - der Mindestlohn erhöht wird. In anderen Ländern finden sich zudem inhaltliche Kriterien für die Entwicklung des Mindestlohns. So wird z. B. in Belgien und Luxemburg der Mindestlohn regelmäßig an die Preissteigerungsraten angepasst, während in Frankreich und den Niederlanden darüber hinaus auch die durchschnittlichen allgemeinen Lohnzuwächse als Richtschnur für die Erhöhung des Mindestlohns dienen (Schulten et al. 2006, S. 18).

\subsection{VORSCHLAG DER RHEINLAND- PFÄLZISCHEN LANDESREGIERUNG}

In Deutschland wurde im Herbst 2007 von der Landesregierung von RheinlandPfalz (2007) ein Entwurf für ein umfassendes Mindestlohngesetz vorgelegt. Obwohl der Gesetzentwurf im Bundesrat von der Mehrheit der CDU-regierten Bundesländer abgelehnt wurde, existiert damit auch hierzulande erstmals ein konkreter Vorschlag für die Einführung und Entwicklung eines allgemeinen gesetzlichen Mindestlohns. Vorgesehen ist, für ganz Deutschland eine unterste Grenze des Arbeitsentgeltes festzulegen, die „vollzeitbeschäftigten Arbeitnehmerinnen und Arbeitnehmern ein ihre Existenz sicherndes Einkommen gewährleisten und eine angemessene Teilhabe am gesellschaftlichen Leben ermöglichen" soll (\$1). Alle Arbeitgeber sind verpflichtet, mindestens den Mindestlohn, der als Bruttoarbeitsentgelt pro Zeitstunde festgelegt wird, zu zahlen (\$2).

$\mathrm{Zu}$ Festsetzung und Anpassung des allgemeinen Mindestlohns soll nach britischem Vorbild eine Mindestlohnkommission eingerichtet werden, die beim Bundesministerium für Arbeit und Soziales (BMAS) angesiedelt ist und sich aus jeweils drei Repräsentanten der Arbeitnehmer und Arbeitgeber sowie drei weiteren sachverständigen Personen zusammensetzt (\$3). Die Mindestlohnkommission soll jeweils bis zum 31. August eines jeden Jahres einen Mindestlohnbetrag vorschlagen ( $\$ 4$, Abs. 1). Stimmt das Bundesministerium für Arbeit und Soziales (BMAS) diesem Vorschlag zu, so kann es den Mindestlohn per Rechtsverordnung bestimmen ( $\$ 4$, Abs. 2). Ist das BMAS mit dem Vorschlag der Mindestlohnkommission nicht einverstanden, so kann es mit Zustimmung der Bundesregierung hiervon abweichende Mindestlohnsätze festlegen ( $\$ 4$, Abs. 3). Ist die Mindestlohn- kommission nicht in der Lage, einen konkreten Vorschlag zu unterbreiten, so kann das BMAS eigenständig per Rechtverordnung einen Mindestlohn vorschreiben $(\$ 4$, Abs. 4).

Der rheinland-pfälzische Mindestlohngesetzentwurf enthält ein im Grundsatz praktikables Modell, das insbesondere die positiven britischen Erfahrungen auf deutsche Verhältnisse zu übertragen sucht. Er formuliert allerdings keine inhaltlichen Kriterien zur Anpassung des Mindestlohns wie z. B. die Entwicklung der Lebenshaltungskosten und/oder die allgemeine Lohnentwicklung. Fraglich ist auch, ob die vorgesehenen Maßnahmen zur Kontrolle des Mindestlohns, die analog zum AEntG von der Zollverwaltung durchgeführt werden sollen $(\$ 5)$, ausreichen. Um eine effektive Kontrolle eines gesetzlichen Mindestlohns sicherzustellen, müssten auch die Kontrollbefugnisse der betrieblichen und gewerkschaftlichen Interessenvertretung gestärkt und z. B. ein gewerkschaftliches Verbandsklagerecht gegen Mindestlohnverstöße eingeräumt werden.

\subsection{MINDESTLOHN UND BESCHÄFTIGUNG}

Die Befürchtung, dass die Einführung eines Mindestlohns zu negativen Beschäftigungseffekten führen würde, ist das Kernargument der Mindestlohngegner. Diese stützen sich auf den wirtschaftswissenschaftlichen Mainstream in Deutschland, der diverse Schreckensszenarien entworfen hat:

An der Spitze findet sich das Institut der deutschen Wirtschaft (IW), das bei Einführung eines gesetzlichen Mindestlohns von $7,50 €$ pro Stunde „bis zu 4 Mio. Arbeitsplätze" bedroht sieht (Hüther 2008). Das Institut für Wirtschaftsforschung in Halle (IWH) konzediert zwar, dass es „weithin unklar" sei, wie sich bei der Einführung eines Mindestlohns „das Arbeitmarktregime anpassen wird“. Dies hält das IWH jedoch nicht davon ab, die etwa $20 \%$ der Beschäftigten in Ostdeutschland, die heute weniger als $7,50 €$ pro Stunde verdienen, als ,potentiell von Arbeitslosigkeit bedroht" einzustufen (Brautzsch/ Schultz 2008). Schließlich hat das ifo Institut Berechnungen angestellt, wonach bei einem Mindestlohn von 7,50€ pro Stunde insgesamt 1,1 Mio. Beschäftigte ihren Arbeitsplatz verlieren würden (Ragnitz/Thum 2008).
Anders als in der öffentlichen Debatte oft suggeriert wird, verfügen die Wirtschaftsinstitute keineswegs über wissenschaftlich gesicherte Nachweise der negativen Beschäftigungswirkungen von Mindestlöhnen. Bei den Aussagen handelt es sich um bloße Hypothesen, die auf bestimmten theoretischen Grundannahmen basieren. Die Grundlage bildet hierbei ein einfaches neoklassisches Arbeitsmarktmodell, wonach der aktuell gezahlte Lohn stets dem Marktlohn entspricht, der wiederum mit der Produktivität des einzelnen Beschäftigten in Einklang steht. Wird dieser Marktlohn nun „künstlich“ durch einen gesetzlichen Mindestlohn erhöht, so übersteigen die Arbeitskosten die Produktivität bestimmter Beschäftigtengruppen und das Unternehmen wird zu Entlassungen gezwungen.

Wie hoch genau die Beschäftigungseffekte ausfallen, hängt in der neoklassischen Arbeitsmarkttheorie von der sogenannten Lohnelastizität der Arbeitsnachfrage ab. Während die Behauptungen von IW und IWH implizit von einer Lohnelastizität von annähernd minus 1,0\% ausgehen, hat das ifo Institut bei seinen Berechnungen explizit eine Lohnelastizität von minus 0,75\% zugrunde gelegt, d.h. dass bei einer Lohnerhöhung von $1 \%$ die Beschäftigung um $0,75 \%$ zurückgeht. Die Annahme einer negativen Lohnelastizität von $0,75 \%$ erscheint allerdings einigermaßen willkürlich, zumal - wie das ifo Institut selbst eingesteht - die in empirischen Studien ermittelte Lohnelastizität mit Werten zwischen minus $0,2 \%$ und minus $2,0 \%$ eine sehr große Schwankungsbreite aufweist, was die Vagheit der Schlussfolgerungen erhöht. Legt man statt des neoklassischen Arbeitsmarktmodells ein keynesianisch orientiertes Modell zugrunde, das kreislauftheoretisch argumentiert und damit auch Nachfrageeffekte von Lohnerhöhungen berücksichtigt, so ergeben sich bei der Einführung eines Mindestlohns in Deutschland sogar positive Beschäftigungseffekte (Bartsch 2007).

Die von der Mehrzahl der deutschen Wirtschaftsforschungsinstitute vertretenen Positionen sind nicht zuletzt auch durch eine höchst einseitige Rezeption der internationalen wirtschaftswissenschaftlichen Debatte geprägt. Den wichtigsten Referenzpunkt bildet hierbei eine umfangreiche Literaturstudie zu den Auswirkungen von Mindestlöhnen auf die Beschäftigung, die von den amerikanischen Autoren Neumark und Wascher (2007) erstellt wur- 
de. ${ }^{2}$ Sie kommen zu dem Ergebnis, dass knapp zwei Drittel der insgesamt 102 von ihnen ausgewerteten Untersuchungen $\mathrm{zu}$ Mindestlöhnen negative Einflüsse auf die Beschäftigung ausweisen. Wie die Autoren selber einschränkend eingestehen, handelt es sich hierbei jedoch keineswegs immer um mit statistischer Signifikanz nachgewiesene Beschäftigungseffekte (ebd., S. 121). Hinzu kommt, dass mehr als $40 \%$ der berücksichtigten Studien lediglich die Auswirkungen auf jugendliche Arbeitnehmer untersuchen und damit das Gesamtbild deutlich verzerren. Besonders fragwürdig ist schließlich die von Neumark und Wascher aufgestellte Behauptung, wonach insbesondere die „wissenschaftlich anspruchsvolleren Studien“ mit großer Mehrheit negative Beschäftigungseffekte feststellen würden (ebd.). Davon abgesehen, dass allein die Hälfte der als wissenschaftlich besonders anspruchsvoll klassifizierten Studien aus den USA von Neumark und Wascher selber durchgeführt wurden, benennen sie keine nachvollziehbaren Kriterien für ihre Auswahl.

Immerhin müssen auch Neumark und Wascher (2007) eingestehen, dass es innerhalb der internationalen Mindestlohnforschung keinen Konsens mehr gibt und ein relevanter Anteil von Studien die traditionelle neoklassische Betrachtung infrage stellt. Hierzu gehören insbesondere diejenigen Arbeiten, die im Sinne eines ,natürlichen Experimentes" die konkreten Auswirkungen von Mindestlohnerhöhungen untersucht haben. In den USA geht heute die Mehrzahl der Wirtschaftswissenschaftler davon aus, dass mit den Mindestlöhnen keine oder nur sehr geringe negative Beschäftigungseffekte verbunden seien. In Europa sind insbesondere die Erfahrungen aus Großbritannien sehr aufschlussreich, da hier erst 1999 ein gesetzlicher Mindestlohn eingeführt wurde, was einem natürlichen Großexperiment gleichkommt. Übereinstimmend gelangen die zahlreichen britischen Studien zu dem Ergebnis, dass die Einführung und spätere Erhöhung des Mindestlohns in Großbritannien gesamtwirtschaftlich keine negativen Auswirkungen auf die Beschäftigung hatte (Metcalf 2007). Schließlich konnte auch für Deutschland jüngst eine Studie des Institutes für Arbeitsmarkt und Berufsforschung (IAB) nachweisen, dass von dem seit 1997 existierenden Mindestlohn in der Bauindustrie keine signifikant negativen Beschäftigungseffekte ausgegangen sind (König/Möller 2007).

\begin{tabular}{|c|c|c|c|c|}
\hline & Hartz IV & $\begin{array}{l}\text { Pfändungs- } \\
\text { freigrenze }\end{array}$ & $\begin{array}{l}\text { Armutslohn- } \\
\text { schwelle }\end{array}$ & $\begin{array}{l}\text { Europäische } \\
\text { Sozialcharta }\end{array}$ \\
\hline Definition & $\begin{array}{l}\text { Regelsatz, } \\
\text { Kosten der } \\
\text { Unterkunft und } \\
\text { Freibeträge }\end{array}$ & $\begin{array}{l}\text { Gesetzlich } \\
\text { definierter } \\
\text { Einkommens } \\
\text { betrag, der einem } \\
\text { verschuldeten } \\
\text { Arbeitnehmer } \\
\text { nicht gepfändet } \\
\text { werden darf }\end{array}$ & $\begin{array}{l}50 \% \text { des Brutto- } \\
\text { Durchschnitts- } \\
\text { lohns }^{3}\end{array}$ & $\begin{array}{l}\text { Recht auf ein } \\
\text { gerechtes } \\
\text { Arbeitsentgelt, } \\
\text { (Artikel } 4 \text { ) } \\
60 \% \text { des Netto- } \\
\text { Durchschnittslohns } 4\end{array}$ \\
\hline Referenzwert & $\begin{array}{l}\text { monatlich } \\
987,00 € \text { netto }\end{array}$ & $\begin{array}{l}\text { monatlich } \\
989,99 € \text { netto }\end{array}$ & $\begin{array}{l}\text { pro Stunde } \\
10,00 € \text { brutto }\end{array}$ & $\begin{array}{l}\text { pro Stunde } \\
7,79 € \text { netto }\end{array}$ \\
\hline $\begin{array}{l}\text { Brutto- } \\
\text { Mindestlohn } \\
\text { pro Monat }{ }^{2}\end{array}$ & $1.360 €$ & $1.367 €$ & $1.670 €$ & $2.006 €$ \\
\hline $\begin{array}{l}\text { Brutto- } \\
\text { Mindestlohn } \\
\text { pro Stunde }\end{array}$ & $8,14 €$ & $8,19 €$ & $10,00 €$ & $12,01 €$ \\
\hline \multicolumn{5}{|c|}{$\begin{array}{l}\text { 1) bezogen auf einen Alleinstehenden, geb. 1960, keine Kinder, gesetzliche Krankenkasse mit 13,8\%, Kirchensteuer, Bundesland } \\
\text { Berlin, Steuerklasse I (2008) } \\
\text { 2) bezogen auf eine } 38,5 \text { Stunden Woche (= } 167 \text { Stunden pro Monat) } \\
\text { 3) } 2007 \text { lag der durchschnittliche Bruttostundenlohn bei } 20,00 € \\
\text { 4) } 2007 \text { lag der durchschnittliche Nettostundenlohn bei } 12,98 € \\
\text { Quelle: Statistisches Bundesamt; Berechnungen des WSI. }\end{array}$} \\
\hline
\end{tabular}

Die internationale Forschung zu Mindestlöhnen macht deutlich, dass das hierzulande von den meisten Wirtschaftsinstituten immer noch präferierte neoklassische Arbeitsmarktmodell nicht in der Lage ist, die Wirklichkeit adäquat zu beschreiben. Insbesondere die britischen Erfahrungen haben gezeigt, dass die Einführung von Mindestlöhnen vor allem zu einer Reduzierung der Profite geführt hat, ohne dass hieraus Beschäftigungsverluste resultierten (Draca et al. 2007). Dies deutet darauf hin, dass vor der Einführung des Mindestlohns viele Unternehmen aufgrund ihrer Marktmacht Löhne gezahlt haben, die unterhalb des neoklassischen Marktlohns lagen und damit „nicht marktgerechte“ Monopolgewinne ermöglicht haben. Dementsprechend werden in den internationalen Wirtschaftswissenschaften immer öfter "monopsonistische" Arbeitsmarktmodelle verwendet, die gegenüber den neoklassischen Vorstellungen ein deutlich realistischeres Bild des Arbeitsmarktes zeichnen (Manning 2003). Vor diesem Hintergrund stellt der gesetzliche Mindestlohn in erster Line ein politisches Instrument dar, um die Marktmacht der Unternehmen zu begrenzen und damit überhaupt erst die Voraussetzung für „marktgerechte“ Löhne zu schaffen.

\subsection{ORIENTIERUNGSGRÖßEN FÜR EINEN GESETZLICHEN MINDESTLOHN}

Bei der Einführung eines gesetzlichen Mindestlohnes in Deutschland stellt sich schließlich die Frage, welche Höhe dafür angemessen ist. In der öffentlichen Diskussion wird hierbei implizit immer wieder der normative Maßstab formuliert, demzufolge ein Vollzeitbeschäftigter von seiner Erwerbsarbeit ohne zusätzliche Unterstützung „leben können muss“. Dies verdeutlicht, dass der Mindestlohn für ein bestimmtes soziokulturelles Existenzminimum steht. Die Frage, wie ein solches soziokulturelles Existenzminimum bestimmt wird, ist wiederum Gegenstand gesellschaftlicher Debatten und Auseinandersetzungen (Tabelle 2).

Eine weit verbreitete Position geht davon aus, dass mit der Grundsicherung nach Hartz IV ein bestimmtes soziokulturelles Existenzminimum festgelegt ist, das implizit bereits heute einen Mindestlohn definiert. Einige Wirtschaftswissenschaftler, wie der Vorsitzende des Sachverständigenrates Bert Rürup (2007), haben sich explizit dafür ausgesprochen, Hartz IV als Orientierungsmarke zu nehmen und plädieren für die Einführung eines gesetzlichen Mindestlohns von 4,50€ pro Stunde. Dies entspricht in der Tat ungefähr dem nach Hartz IV gezahlten Regelsatz plus der Kosten für die Unterkunft eines Alleinstehenden. Al-

2 Neumark und Wascher sind in den USA als langjährige Mindestlohnkritiker bekannt und haben in der Vergangenheit u.a. mehrere Studien für das Employment Policies Institute durchgeführt, bei dem es sich um einen von der amerikanischen Hotel- und Gastronomiebranche gesponserten Think Tank handelt, der sich auf Studien zur Kritik von Mindestlöhnen spezialisiert hat. 
lerdings wird von Rürup übersehen, dass ein Erwerbtätiger normalerweise einen höheren Bedarf hat als ein Nicht-Erwerbstätiger, schon allein, um die mit der Erwerbsarbeit verbundenen Kosten (z. B. Fahrgeld, Arbeitskleidung usw.) zu kompensieren. Dementsprechend gelten für einen erwerbstätigen Hartz IV-Empfänger nach dem Sozialgesetzbuch (SGB II, $\$ 11$ und $\$ 30$ ) bestimmte Freibeträge, die nicht auf die Transferleistungen angerechnet werden. Bei voller Ausschöpfung aller Freibeträge ergibt sich für einen alleinstehenden erwerbstätigen Hartz-IV-Empfänger ein monatlicher Netto-Bedarf von $987 €$, was brutto bei einer 38,5-Stunden Woche einem Stundenlohn von $8,14 €$ entspricht.

Neben Hartz IV könnte die sogenannte Pfändungsfreigrenze eine Orientierungsgröße zur Festsetzung eines gesetzlichen Mindestlohns bilden. Der Gesetzgeber hat festgelegt, dass einem alleinstehenden verschuldeten Arbeitnehmer das Einkommen erst ab einer Grenze von derzeit 989,99 € netto gepfändet werden darf. Umgerechnet auf einen Bruttostundenlohn ergibt sich hieraus ein Betrag von 8,19€, der ungefähr dem nach Hartz IV berechneten Mindestlohn entspricht.

Neben der Orientierung auf ein soziokulturelles Existenzminimum lassen sich darüber hinaus auch weitergehende Kriterien benennen, die den Mindestlohn ins Verhältnis zum allgemeinen Lohngefüge in Deutschland setzen. In Anlehnung an die internationale Armutsforschung kann eine „Armutslohnschwelle“ bestimmt werden, die bei $50 \%$ des Brutto-Durchschnittslohns liegt. Im Jahr 2007 lag der BruttoDurchschnittslohn in Deutschland bei $20,00 €$ pro Stunde, sodass sich hieraus eine Mindestlohnhöhe von $10,00 €$ pro Stunde ergeben würde.

In eine ähnliche Richtung zielt schließlich das in der von Deutschland ratifizierten Europäischen Sozialcharta des Europarates festgeschriebene „Recht auf ein gerechtes Entgelt“ (Artikel 4), das ,allen Arbeitnehmern ... und ihren Familien einen angemessenen Lebensstandard sichern" soll. Um die Umsetzung dieses sozialen Grundrechts operationalisieren zu können, ist vom Europarat ein Schwellenwert definiert worden, der bei $60 \%$ des Netto-
Durchschnittseinkommens liegt (Lörcher 2006, S. 222). In Deutschland läge ein solcher Schwellenwert bei netto 7,79 € pro Stunde, was brutto einem Stundenlohn von $12 €$ entsprechen würde. Angesicht des real existierenden Armutslohnsektors ist Deutschland vom Europarat mehrfach wegen des Verstoßes gegen die Europäische Sozialcharta kritisiert worden (zuletzt: Council of Europe 2007).

Soll ein gesetzlicher Mindestlohn - wie im Mindestlohngesetzentwurf von Rheinland-Pfalz (2007, S.6) gefordert - dazu beitragen, auch in Deutschland das „Recht auf ein gerechtes Entgelt" umzusetzen, so müssten die unteren Lohngruppen erheblich angehoben werden. Die Orientierung am soziokulturellen Existenzminimum müsste in Deutschland zu einem gesetzlichen Mindestlohn führen, der mit einem Betrag zwischen 8 und $9 €$ pro Stunde den Mindestlöhnen in anderen westeuropäischen Staaten entspricht. Die aktuelle gewerkschaftliche Forderung nach einem gesetzlichen Mindestlohn von 7,50 pro Stunde als Einstieg ist vor diesem Hintergrund keineswegs zu hoch gegriffen. 
Bartsch, K. (2007): Gesamtwirtschaftliche Wirkungen der Einführung eines gesetzlichen Mindestlohnes in Deutschland, in: WSI-Mitteilungen 11, S. 589-595

Bispinck, R./WSI-Tarifarchiv (2007): Unterste Tarifvergütungen. Ausgewählte Tarifbereiche mit unterster Tarifgruppe und Laufzeitende der Vergütungstarifverträge. Reihe: Elemente qualitativer Tarifpolitik 63, Düsseldorf

Bundesministerium für Arbeit und Soziales (BMAS) (2008a): Referentenentwurf: Gesetz zur Gewährleistung angemessener Arbeitsbedingungen für grenzüberschreitend entsandte und für regelmäßig im Inland beschäftigte Arbeitnehmer und Arbeitnehmerinnen (Arbeitnehmer-Entsendegesetz - AEntG) vom 11.01.

Bundesministerium für Arbeit und Soziales (BMAS) (2008b): Referentenentwurf für ein erstes Gesetz zur Änderung des Gesetzes über die Festsetzung von Mindestarbeitsbedingungen (Mindestarbeitsbedingungen-Änderungsgesetz - 1. MiArbGÄndG) vom 10.01.

Brautzsch, H.-U./Schultz, B. (2008): Aktuelle Trends: Jeder fünfte abhängig Beschäftigte im Osten verdient weniger als 7,50 Euro je Stunde, in: Wirtschaft im Wandel 2, S. 59

Burgess, P. (2006): Der gesetzliche Mindestlohn in Großbritannien, in: Schulten, T. et al. (Hrsg.): Mindestlöhne in Europa, Hamburg, S. 31-55 Council of Europe (2007): European Social Charter, Conclusions XVIII-2 on Germany by the European Committee of Social Rights, December online: www.coe.int/t/e/human_rights/esc/3_reporting_procedure/ 2_recent_conclusions/1_by_state/GermanyXVIII2_en.pdf Draca, M./Machin, S./Van Reenen, J. (2006): Minimum Wages and Firm Profitability, CEP Discussion Paper 715, February

Hüther, M. (2008): 4 Millionen Jobs in Gefahr, in: Bild am Sonntag vom 13. Januar, S. 5
Kalina, T./Weinkopf, C. (2008): Weitere Zunahme der Niedriglohnbeschäftigung, IAQ-Report 1

König, M./Möller, J. (2007): Mindestlohneffekte des Entsendegesetzes? Eine Mikrodatenanalyse für die deutsche Bauwirtschaft, IAB Discussion Paper 30

Landesregierung Rheinland-Pfalz (2007): Entwurf eines Gesetzes über die Festsetzung des Mindestlohns (Mindestlohngesetz - MindLohnG), Bundesrat Drucksache 622/07 vom 04.09.

Lörcher, K. (2006): Das Recht auf angemessenes Arbeitsentgelt nach der Europäischen Sozialcharta, in: Sterkel, G. et al. (Hrsg.): Mindestlöhne gegen Lohndumping, Hamburg, S. 216-228

Manning, A. (2003): Monopsony in Motion, Imperfect Competition in Labour Markets, Princeton

Metcalf, D. (2007): Why Has the British National Minimum Wage Had Little or No Impact on Employment?, CEP Discussion Paper 781, April Neumark, D./Wascher, W. (2007): Minimum Wages and Employment, IZA Discussion Paper 2570

Peter, G. (2004): Mindestarbeitsbedingungsgesetz, in: Peter, G. et al.: Die Sicherung tariflicher Mindeststandards, Baden-Baden, S. 83-103

Ragnitz, J./Thum, M. (2008): Beschäftigungswirkungen von Mindestlöhnen - eine Erläuterung zu den Berechnungen des ifo Institutes, in: ifo Schnelldienst 1, S. 16-20

Rürup, B. (2007): 4,50 Euro pro Stunde. Warum wir einen gesetzlichen Mindestlohn brauchen, in: Die Zeit 47 vom 15. November Schulten, T./Bispinck, R./Schäfer, C. (Hrsg.) (2006): Mindestlöhne in Europa, Hamburg 